\title{
Enhanced 6LoWPAN Ad Hoc Routing for G3-PLC
}

\author{
Kaveh Razazian, Afshin Niktash, Victor Loginov \\ Smart Grid Solutions \\ Maxim Integrated \\ Irvine, CA, USA
}

\author{
Javad Yazdani \\ University of Central Lancashire \\ Lancashire, UK
}

\begin{abstract}
G3-PLC is a powerline communication standard employing OFDM technology for the physical layer. The Medium Access Control (MAC) layer is derived from IEEE 802.15.4 as an interface between Logical Link Control (LLC) and the physical layer. It adopted Low-Power Wireless Personal Area Network (6LoWPAN) specification to facilitate IPv6 interaction at the network layer. Due to hostile power line environment, 6LoWPAN Ad hoc protocol (LOAD) is used as an efficient routing method over varying network topologies and link conditions. Since LOAD is originally developed for wireless networks, it does not consider some specific channel characteristics of power line networks in its routing strategy. This paper describes unique enhancements to LOAD routing tailored to power line communications to address issues such as asymmetrical routing, to minimize the number of control messages and to handle the ghost node issue.
\end{abstract}

Keywords- G3-PLC; Routing; LOAD; LOADng; 6LoWPAN; Ad-hoc; AODV; AODVjr

\section{INTRODUCTION}

The use of power line distribution grid for data communication has gained heightened interest over the past several years. It is well known that a power line channel is far from being an ideal channel for data transmission. Power line channel characteristics and parameters typically vary with frequency, location, time and the type of equipment connected to the channel. While the high-frequency regions above $1 \mathrm{MHz}$ exhibit significant multipath frequency selective fading accompanied by severe attenuation on distances above several $100 \mathrm{ft}$, the lower frequency regions from $20 \mathrm{kHz}$ to $500 \mathrm{kHz}$ are especially susceptible to narrowband interference and impulsive noise [1].

Due to significant interest in utilizing the low frequency regions for data communication on power lines, several standards have been introduced since 2010 such as G3-PLC [2], PRIME [3], ITU G.9955/9956[4] and P1901.2 [5]. G3PLC is one of the specifications which uses OFDM technology employing concatenation of a Reed-Solomon(RS) encoder with a $1 / 2$ rate convolutional encoder, followed by a $4: 1$ repetition coding block for Robust mode (ROBO) cascaded with a twodimensional time and frequency interleaving. G3-PLC system supports three different modulations, DBPSK, DQPSK, and D8PSK [6]. Furthermore MAC layer in G3-PLC is derived from IEEE 802.15.4 as an interface between Logical Link Control (LLC) and the physical layer to regulate the usage of the medium with CSMA/CA, to support 16 and 64 bits local addressing, to provide positive and negative feedback to a received data packet from an originator in form of ACK (positive acknowledgment) and NACK (negative acknowledgement) and to perform packet fragmentation /assembly.

G3-PLC also uses IPv6 addressing at the network layer and adopted 6LoWPAN technology to facilitate IPv6 adoption at low-rate networks. 6LoWPAN allows power line network similar to a wireless network to incorporate IPv6 in embedded equipment for seamless connection of MAC layer and network layer to achieve the header compression, fragmentation and assembly [7].

In spite of the superior performance of G3-PLC physical layer in a hostile power line environment, it is essential to select an efficient routing protocol over 6LoWPAN which can quickly adapt to varying network topology and link condition. LOAD is a simplified on-demand routing protocol based on Ad-hoc on Demand distance Vector routing (AODV) and is one the most promising routing scheme which has been drafted within the 6LoWPAN working group in IETF [8] and is used as the base for G3-PLC and ITU G9956 specification.

Ad-hoc routing is simply a reactive mesh routing which finds a route to a destination by broadcasting Route Request (RREQ) messages per demand and each node learns about its neighbor node through which it can connect to the destination. There has been a lot of research conducted on modeling and simulation of Ad-Hoc routing mainly for the wireless network [7]-[12] over the past several years. In particular, Perkins et al. introduced AODV algorithm to establish routes in mobile nodes without the requirement of any centralized access point or existing infrastructure[9]. Perkins' approach eliminates broadcasting periodic route advertisements to keep the network up to date with new routing tables. However the node intending to communicate would broadcast a RREQ message throughout the network until desired destination is reached. Upon reception of RREQ at destination, the destination node should unicast a Route Reply (RREP) message back to the source node assuring a symmetrical link between source and destination [10]. Moreover, should a node detect a broken link 
a Route Error (RERR) is broadcasted to initiate a new source discovery process [10].

As low-power wireless applications such as sensors network expands, these large several hundred sensor nodes need to gather information in scenarios that fixed infrastructure cannot be realized [12]. This has been the motivation to propose a simplified AODV to make the protocol more efficient for low-power devices with limited bandwidth and processing and memory storage capabilities such as AODVjr [13], NST-AODV [14] and various versions of AODVjr algorithm [15]-[17]. AODVjr presented by Chakeres et al. is one of the earliest AODV shortened version which significantly reduces the implementation complexity and some of its routing strategies have also been used in drafting LOAD specification. In particular AVODjr does not use the sequence number since only the destination node can reply to a RREQ [16]. The routes in AVODjr protocol are bidirectional. When a route breaks the source stops receiving any massages from destination. After sometime the source detects a broken link and initiates a RREQ if the route is requested [17]. Therefore, there is no need for hello massages, RERR and precursor list.

Since all the routing models described above including LOAD are designed for wireless networks, they do not consider unique channel characteristics of power line in their routing strategy. The authors' main contribution in this paper is to construct enhancements tailored to power line communication to LOAD routing for G3-PLC in order to handle asymmetrical routing, to minimize number of control messages and to address the ghost node scenario. .

The rest of the paper is organized as follows: Section II gives an overview of LOAD routing algorithm, followed by enhancements to LOAD routing for power line communication in section III. Section IV summarizes the on-going enhancements to LOAD-NG in IETF working group. A test platform to explore routing enhancements is presented in section $\mathrm{V}$, followed by a conclusion in section VI.

\section{LOAD ROUTING PROTOCOL}

The 6LoWPAN Ad hoc Routing Protocol (LOAD) [8] is a simplified form of AODV [18] for 6LoWPAN. As a reactive protocol, LOAD operates on adaptation layer creating a mesh network topology underneath IPv6 network layer. For the IPv6 layer, the 6LoWPAN adaptation layer is considered a single link.

LOAD is designed to find an optimized route with minimum Route Cost (RC) between two nodes in a network by generating and forwarding broadcast RREQ messages towards the destination during a discovery period. The optimum discovered route is then examined and being communicated to the originator by generating a unicast RREP message at the destination and forwarding it to the originator.

While LOAD supports the use of both the EUI-64 and the 16 bit short addressing for routing, G3-PLC only allows the latter to be used as a routable address and limits the use of EUI64 addressing for bootstrapping or direct communication to a neighbor. In order to establish and maintain a route, each node is required to accommodate two tables:

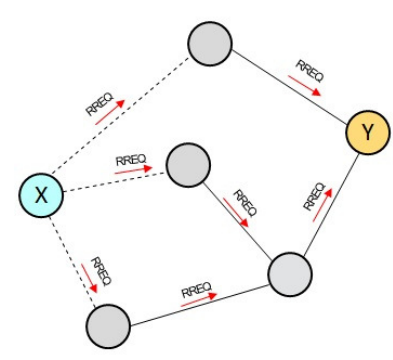

(a)

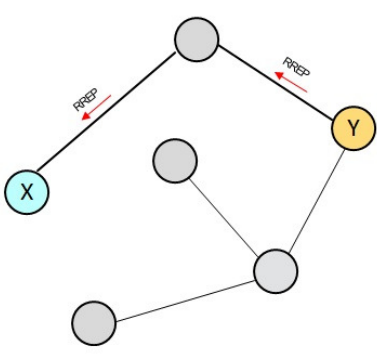

(b)
Figure 1. RREQ and RREP

- Routing Table which includes Destination Address, Next Hop Address to the destination, Status of a route and Life Time of a route before being expired.

- Route Request Table which is maintained during the route discovery to keep track of RREQ messages. It includes $R R E Q I D$ which is a sequence number to uniquely identify a RREQ, Originator Address of a RREQ, Forward Route Cost from the originator to the current node, Reverse Route Cost from destination to current node and Valid Time of the entry before being expired.

Following messages are used to establish a route:

- RREQ is a broadcast message originated by the source node and forwarded by middle nodes to the destination. The main fields of the message include: Route Cost which is the accumulated Link Cost from the originator, RREQ ID, WL which is the number of Weak Links and Originator and Destination Addresses.

- RREP is a unicast message originated by the destination node and forwarded by intermediate nodes along the discovered route to the source. The message includes following main fields: Route Cost which is the accumulated Link Costs from the destination, $R R E Q$ $I D, W L$ and Originator and Destination Addresses.

- RERR is a unicast message identifying an error in route discovery and is originated by the intermediate node not being able to repair a link and sent back to the final destination of discovery. The fields of the message include an Error Code and Destination Address of the unreachable node.

In order to perform a route discovery, a source node originates a RREQ message with an incremental RREQ ID and broadcasts it to its neighbors. Every intermediate node that receives the RREQ message should add it to its RREQ Table and rebroadcast it, if another copy of the same RREQ identified by its RREQ ID and Originator Address is not found in its RREQ Table. The RC and WL should be updated in rebroadcasted RREQ and a route to the originator needs to be added to the node's Routing Table. Fig. 1 shows this procedure.

When the destination receives a RREQ, it should be discarded if any other RREQs from the same originator with 
the same RREQ ID with a better forward RC (or $<$ RC,WL $>$ tuple) is found in Route Request Table. Otherwise the destination updates the Route Request Table with this new RREQ, updates Routing Table, generates a unicast RREP and sends it along the route back to the source node.

The RREP is forwarded back to the source node. The WL and RC fields are updated by each intermediate node. In order to limit the forwarding of multiple RREPs, an intermediate node only forwards the RREP if it can find an entry from the same originator with the same RREQ ID with worse Reverse $\mathrm{RC}$. A route to the destination is also added to the Routing Table. Once the originator receives the RREP, it updates its Routing Table with the route to the destination.

If an intermediate node is unable to forward a RREP, it may initiate a Route Repair to find an alternative route to the source. The failure can be reported back to the final destination node using a unicast RERR message. All other nodes forwarding RERR back to the final destination also update their Routing Table by removing the originator node from the table.

\section{ROUTING ENHANCEMENTS}

Since power line channel can be very dynamic and time varying, a node contributing to a route can be out of reach from time to time. Therefore LOAD routing has to be launched frequently to find an alternative route. In G3-PLC broadcast packets are sent in ROBO mode with DBPSK modulation and repetition factor of 4 without any acknowledgement. These packets are longer in time compared to other modulations. Therefore broadcast of RREQs can keep the shared channel between several nodes busy for duration of route discovery which could be significant. During this busy time, normal data communication is not possible. Therefore is it very important to include features to create optimally selected robust and bidirectional routes to avoid frequent route discoveries and to limit the flooding of the network with broadcasting RREQs.

Another contributing factor to the overall stability as well as throughput of the network is minimizing the number of unnecessary logical hops where nodes are physically neighbors.

Following enhancements are introduced to address above limitations to further improve LOAD performance when applied to power line communication.

\section{A. Asymmetrical Routes}

In power line communication, it is frequently observed that a link between two nodes becomes asymmetrical, meaning that the quality of the channel is significantly different depending on the communication direction. For example, the receiver SNR can be very high for sending packets from node A to node $\mathrm{B}$ and very low for sending packets from node B to node A. This results in different optimum modulation in forward and reverse link to establish a robust communication.

In G3-PLC, each unicast packet to a neighbor is responded with an acknowledgment packet (ACK). Therefore, in order to have a stable data communication from one node to another one, both forward and reverse channels along the selected route should be robust enough regardless of direction.
LOAD algorithm does not specifically propose any method to calculate the Link Cost between nodes. In G3-PLC [4], a mathematical formula is suggested to compute the Link Cost based on several parameters, including Modulation (MOD) and Link Quality Indicator (LQI) as

$$
\begin{aligned}
\text { Link Cost }= & K_{r} \cdot \operatorname{MOD}_{K r}+K_{m} \cdot M O D_{K m}+ \\
& K_{c} \cdot \operatorname{Norm}\left(N_{\text {tones }}\right)+K_{q} \cdot \operatorname{Norm}(L Q I)+ \\
& K_{h}+K_{r t} \cdot N_{r} / \max \left(N_{r}\right),
\end{aligned}
$$

where

$$
\operatorname{Norm}(x)=(\max (x)-x) / \max (x),
$$

$M O D_{K m}$ is the modulation (MOD), $N_{\text {tones }}$ is the number of tones, $N_{r}$ is number of routes in Routing Table, $K_{r}, K_{m}, K_{c}, K_{q}$, $K_{h}$ and $K_{r t}$ are weighting coefficients. $M O D_{K r}$ is set for ROBO modulation to further deemphasize this type of links.

A node is capable of calculating the forward LQI and Modulation of a received RREQ packet. However, the reverse channel LQI and MOD cannot be determined from RREQ. There is also no provision in LOAD to allow communicating the reverse channel quality to an intermediate node to take into account in its Link Cost calculation.

In G3, each node is equipped with a Neighbor Table (NT). In node $\mathrm{B}$, the $\mathrm{NT}$ entry associated with node $\mathrm{A}$ includes the reverse $\mathrm{LQI}^{\mathrm{rev}}$ and $\mathrm{MOD}^{\mathrm{rev}}\left(\mathrm{LQI}_{\mathrm{B}->\mathrm{A}}\right.$ and $\left.\mathrm{MOD}_{\mathrm{B}->\mathrm{A}}\right)$ collected as part of channel estimation the first time $\mathrm{B}$ tried to communicate with A and periodically updated afterward during communication. The channel estimation uses tonemap request/response to measure/communicate the reverse channel information to acquire $\mathrm{LQI}^{\mathrm{rev}}$ and $\mathrm{MOD}^{\text {rev }}$ or their best available estimates. Hence, the Link Cost is computed by using $\mathrm{LQI}^{\mathrm{rev}}, \mathrm{LQI}^{\mathrm{for}}, \mathrm{MOD}^{\text {rev }}$ and $\mathrm{MOD}^{\text {for }}$. Once route discovery is complete, both source and destination will add the same route to their Routing Table. Therefore it is important to establish a bidirectional and high quality route over an asymmetrical channel to avoid frequent route discoveries.

\section{B. Forwarding Multiple RREQs}

In LOAD algorithm, if an intermediate node finds the same RREQ ID from the same originator in its Route Request Table, it does not rebroadcast it. This means that the first RREQ arrived at any intermediate node is assumed to have the better route cost from the originator to that intermediate node compared to the RREQs received later. This is not necessarily true as a route request from a better route might have been delayed in some nodes due to the processing load or channel access and not because of a worse channel condition. While this approach reduces the number of broadcast RREQ, it does not provide the optimum route.

In order to address this issue, late RREQs are also rebroadcasted, only if their associated route cost is significantly lower than the last rebroadcasted one. This approach improves the route optimization while still trying to reduce the number of RREQ broadcasts if they are not arrived from better routes.

\section{Minimizing Sending RREPS}

The destination node generates a RREP in response to every RREQ if its route cost is lower than the previously 


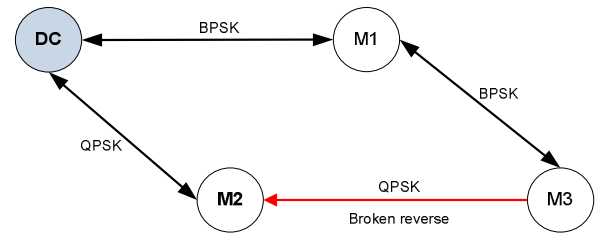

Figure 2. Repairing a broken link

received RREQs from the same source with same RREQ ID. This results in sending multiple RREP through multiple routes. The source needs to wait for some time to make sure that there is no better RREP in transit. This could result in increased traffic of unnecessary RREPs in a typically large power line network.

In order to reduce the RREPs traffic, the waiting period in source to collect all RREPs is moved to the destination. After receiving the first RREQ, the destination will wait for a predefined period to make sure no other RREQ with a better route cost is in transit. At the end of this wait period, the best route is chosen and a RREP associated with that route is generated and sent towards the source.

\section{Beacon Flooding upon Network Powerup}

In G3-PLC bootstrapping procedure, a new device sends a beacon to all neighbors to find a neighbor node which is already associated to the network to act as a forwarder during the association and authentication. All neighbor nodes in the network respond to the beacon with a beacon response. In a typical metering application, several hundred meters can be connected to the same server over the shared channel to create a power line network. After power-up of all devices (e.g. recovery after a black out), all meters start sending beacons while only few devices are already associated and are able to respond. As more devices are associated to the network, more beacon responses are sent in response to a beacon, making the network more congested with more collisions and hence the chance of successful association of new meters decreases. To alleviate this problem, a random delay is defined during the power-up procedure of a node to avoid back-to-back retry if an association attempt failed.

\section{E. Ghost Node}

A ghost (or orphan) node is referred to a device that had been successfully associated to a network and had a very good communication with the server (very low RC) previously. However, due to dynamic changes in the behavior of power line network, the bidirectional communication is no longer possible and the node is not aware of this change. In other words, the device still thinks that it has a perfect route to the server. Since the typical metering application requires infrequent access to a meter, this condition may exist for some time before the meter or server notices the failure in the link. During this period, the ghost meter always responds to any beacon request from a new associating device which may cause a series of repeated failures of association procedure for that device.
To alleviate this problem, a Route Cost to Coordinator (server) field is added to the payload of the beacon response (RC_Coord). Once the first association through a ghost meter fails, a very high value of RC_Coord is reported in any future beacon responses of the ghost meter, indicating that although the meter is part of the network, but is does not currently have a sustainable communication link to the server. The associating meter would use this information to avoid selecting the ghost node for next attempts.

\section{F. Link Repair}

This feature is very accommodating in improving the routing performance especially in asymmetrical routes with unreliable or broken reverse links. RREQ is a broadcast packet and does not require any ACK. If the reverse Link Cost is not known, a node may calculate the RC based on the forward Link Cost and rebroadcast the RREQ. This route can be selected by the destination as the optimum route and the RREP can be dispatched along this route.

Fig. 2 shows an example where the RREP is failed to be forwarded from M2 to M3 during a route discovery originated for M3 to destination DC. Since M3 never receives any RREP, the route discovery fails. Furthermore, all subsequent route discovery attempts result in the same behavior and M3 cannot find a route to DC while an alternative route exists.

The Local Link repair method supported by LOAD can address this issue. A second RREQ originated from M2 is generated as soon as RREP from M2 to M3 fails. This new RREQ, specified by its Local Repair (R) flag set to 1 is dispatched to find an alternative route to $M 3$. DC receives this repair RREQ and rebroadcast it to be finally delivered to M3 through M1. M3 responds back with a RREP to M2 and finally the original RREQ is sent along this repaired route from M2 to M3 and the new route DC-M1-M3 is installed.

Link Repair procedure is very effective in fixing routing issues in unidirectional links in large networks to avoid deadlocks.

\section{LOADng RouTING PROTOCOL}

Developed by several academic and industry leaders, the Lightweight On-demand Ad hoc Distance-vector Routing Protocol - Next Generation (LOADng) [19], is designed to further enhance LOAD with additional features and capabilities. Derived from AODV [20], the basic operation of LOADng is similar to LOAD and includes generation of RREQs by a LOADng Router, forwarding them until they reach the destination, generation of RREPs upon receipt of an RREQ by the indicated destination, and hop-by-hop forwarding of these unicast RREPs towards the originator. It also employs RERR message to report a broken link if a data packet cannot be forwarded. The following highlights the additional features of LOADng:

\section{A. Blacklisting}

The Blacklisted Neighbor Set maintains the address of nodes to which the connection is detected to be unidirectional. More specifically, if a RREP to a neighbor from which a 
RREQ has been received fails, it is been added to the Blacklist. When a neighbor is blacklisted, any RREQ received from that node is dropped. Blacklisting avoids the selection of the same unidirectional link repeatedly after a RREP fails due to a broken reverse link as demonstrated in Fig. 2 and described before.

\section{B. Separate Forward and Reverse Routes}

Using the LOAD algorithm, the routing tables are updated to use the same route for both directions of communication. In other words, at the end of route discovery both the originator and destination will install the same route in their routing tables regardless of the node which initiated the procedure.

LOADng introduces the Routing Set which is a separate structure from Routing Table. Only Routing Sets are updated during route discovery and Routing Tables can selectively use a route form Routing Set. This provides provisions to establish different routes between two nodes based on the initiator of the route discovery and hence to create and use two separate routes between two nodes, A \& B depending on direction of communication: an optimized route for sending packets from $\mathrm{A}$ to $\mathrm{B}$ while having a viable link from $\mathrm{B}$ to $\mathrm{A}$, and an optimized route for sending packets from $B$ to $A$ while having a viable link from A to B.

\section{Extension for Route Cost Calculation}

LOADng allows 16-bit values to be used for route metric while LOAD only allocates 8 bits. This results in a better resolution when comparing route costs and more number of hops to be used when accumulating the Link Costs along a route. Furthermore, using Type-Length-Value (TLV) elements enables protocol extensions to be developed [20].

\section{TEST PlatForm}

In an attempt to replicate some pressing issues of routing observed in field trials, a test platform was created to explore the enhancements in a controlled environment. A series of attenuators (a1 to a6) and relays (R1-R4) and noise source are connected as shown in Fig. 3 to build a variety of network topologies. Various topologies such as unidirectional, asymmetrical and multi-hop connections are modeled by proper selection of attenuators and noise source. Two series of tests were conducted.

In Static Topology tests, a condition was tested by fixing the configuration of attenuators and relays to explore the effect of proposed methods. For example, an asymmetrical route similar to what had been observed when crossing MV-LV transformer was created. In this scenario, a remote node in LV side was trying to communicate directly with a far concentrator (server) in MV side without using a repeater node placed in LV side of the MV-LV transformer, resulting in an unreliable communication. Table I shows a number of test cases. For each test, $100 \mathrm{~K}$ packets were transmitted with maximum packet size of 1280 bytes. Both end devices were set to transmit during the test to flood the network with back-to-back packets and to increase collisions. The packet loss is expected once packets are sent through forwarders due to high number of collisions related to hidden node effect. In another test high level of noise

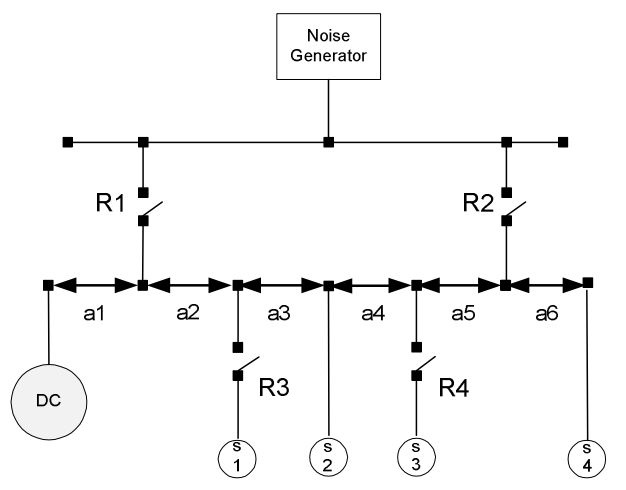

Figure 3. Test Platform Setup

was introduced to the channel and a marginal communication is created. As shown, packet loss increased as a result of poor channel condition creating frequent failed retransmissions and also high collision rates. Nevertheless, the routing protocol was successful in maintaining the link in such a harsh condition.

With Dynamic Topology tests, a script was developed to dynamically change the configuration to simulate the time varying behavior of power line in stressed networks. In this mode, a series of cross-communication and poll-response meter reading tests were defined. By dynamically modifying the network topology while nodes were under high traffic stress, a new route had to be discovered to resume the communication. Table II summarizes the result of a sample Dynamic Topology test. In the first step, two routes were available and meters were communicating through a preferred route. Then the network was abruptly switched to make the route from DC to S2 asymmetrical. Thanks to the routing protocol, the selected route changed quickly. In next step a new device S4 which was trying to associate to the network was introduced. Consequently the longer route was selected as the DC to S3 was asymmetrical. Once $\mathrm{S} 1$ is disconnected, a marginal ROBO channel due to the noise was the only option to establish communication between S4 and DC. Next, by making DC to $\mathrm{S} 3$ a symmetrical route, $\mathrm{S} 4$ prefers to use this route rather than previously selected marginal route.

Table I

\begin{tabular}{|c|c|c|c|}
\hline Description & Noise Source & Interframe Gap & Packet Loss \\
\hline DC \& S1 & R1 \& R2 open & $10 \mathrm{msec}$ & 0 \\
\hline $\begin{array}{c}\text { DC \& S2 } \\
\text { via S1 }\end{array}$ & R1 \& R2 open & $10 \mathrm{msec}$ & $5 \mathrm{e}-5$ \\
\hline $\begin{array}{c}\text { DC \& S2 } \\
\text { via S1 }\end{array}$ & $\begin{array}{c}\text { R1 closed, } \\
\text { marginal ROBO }\end{array}$ & $10 \mathrm{msec}$ & $7 \mathrm{e}-3$ \\
\hline
\end{tabular}

\begin{tabular}{|c|c|c|c|}
\hline \multicolumn{4}{|c|}{ Table II } \\
\hline Description & Route 1 & Route 2 & $\begin{array}{l}\text { Winning } \\
\text { Route }\end{array}$ \\
\hline 1:Two routes & $\mathrm{DC} \Leftrightarrow \mathrm{S} 1 \Leftrightarrow \mathrm{S} 2$ & $\mathrm{DC} \Leftrightarrow \mathrm{S} 3 \Leftrightarrow \mathrm{S} 2$ & Route 2 \\
\hline $\begin{array}{l}\text { 2:Make } \\
\text { asymmetrical }\end{array}$ & $\mathrm{DC} \Leftrightarrow \mathrm{S} 1 \Leftrightarrow \mathrm{S} 2$ & $\mathrm{DC} \Leftrightarrow \mathrm{S} 3 \Leftrightarrow \mathrm{S} 2$ & $\begin{array}{c}\text { Switches to } \\
\text { Route } 1\end{array}$ \\
\hline 3:Associate S4 & $\mathrm{S} 4 \Leftrightarrow \mathrm{S} 3 \Leftarrow \mathrm{DC}$ & $\begin{array}{c}\mathrm{S} 4 \Leftrightarrow \mathrm{S} 2 \Leftrightarrow \mathrm{S} 1 \Leftrightarrow \\
\mathrm{DC}\end{array}$ & Use route2 \\
\hline $\begin{array}{l}\text { 4:Disconnect } \\
\text { S1 }\end{array}$ & $\mathrm{S} 4 \Leftrightarrow \mathrm{S} 2 \Leftrightarrow \mathrm{DC}$ & N/A & $\begin{array}{c}\text { Marginal } \\
\text { ROBO } \\
\text { S2 } \Leftrightarrow \text { DC }\end{array}$ \\
\hline $\begin{array}{l}\text { 5:Make } \\
\text { symmetrical } \\
\text { DC } \Leftrightarrow S 3\end{array}$ & $\mathrm{~S} 4 \Leftrightarrow \mathrm{S} 2 \Leftrightarrow \mathrm{DC}$ & $\mathrm{S} 4 \Leftrightarrow \mathrm{S} 3 \Leftrightarrow \mathrm{DC}$ & Use route 2 \\
\hline
\end{tabular}




\section{CONCLUSION}

Due to the dynamic and time varying condition of power line communication, efficiency of the routing algorithm is of utmost importance to the robustness of the network. Since LOAD protocol was developed targeting wireless mesh networking requirements, a number of enhancements were investigated to tailor it to power line specification. Asymmetrical routes as one of the major contributors to network failure are avoided by using the reverse LQI and modulation with a conservative Link Cost calculation. Other improvements are described to reduce the RREQ and RREP traffic and network flooding and to avoid a dead-lock in establishing a link. The test results showed the effectiveness of the proposed methods in challenging scenarios.

\section{REFERENCES}

[1] K. Razazian, M.Umari, A. Kamalizad, V. Loginov, M. Navid, "G3PLC specification for powerline communication: overview, system simulation and field trial results," IEEE Intl. Symposium on Power Line Communications and Its Applications, 2010.

[2] G3-PLC physical layer specification and G3-PLC MAC Layer specification, G3-PLC Alliance, Aug. 2012.

[3] PRIME Specification, "Draft specification for PoweRline Intelligent Metering Evolution,” Ver. 1.3.6, PRIME Alliance, 2012.

[4] Recommendation ITU-T G.9903 Narrowband orthogonal frequency division multiplexing power line communication transceivers for G3PLC networks, Oct. 2012.

[5] "IEEE P1901.2 draft standard for low frequency (less than $500 \mathrm{kHz}$ ) narrow band power line communications for smart grid applications," IEEE P1901 working group, Oct. 2012.

[6] K. Razazian, M.Umari, A. Kamalizad, "Error correction mechanism in the new G3-PLC specification for powerline communication," IEEE Intl. Symposium on Power Line Communications and Its Applications, 2010.

[7] Xin Ma, Wei Luo, "The analysis of 6LowWPAN technology," Workshop on Comutational Intellegent and Industrial Application, IEEE Trans. on Power Delivery, Vol. 19, No. 3, pp. 1057-1064, 2004.

[8] K. Kim, S. Daniel Park, G. Montenegro,S. Yoo, N. Kushalnagar, "6LoWPAN ad hoc on-demand distance vector routing (LOAD)," http://tools.ietf.org/html/draft-daniel-6lowpan-load-adhoc-routing-03.

[9] C.Perkins, E. Belding-Royer, "Ad-hoc on-demand distance vector routing," Mobile Computing Systems and Applications, pp. 90 - 100, 1999.

[10] G. Lakshmikanth, M.A Gaiwak, P.D Vyavahare, "Simulation based comparative performance analysis of adhoc routing protocols," TENCON IEEE Region 10 Conference, pp. 1-5, 2008.

[11] J.M Chang, T.Y Chi, H.Y Yang, H.C. Chao, "The 6LoWPAN ad-hoc on demand distance vector routing with multi-path scheme," Frontier Computing. Theory, Technologies and Applications, IET International Conference , pp. 204-209, 2010.

[12] S.Gowrishankar, T.G. Basavaraju, SubirKumarSarkar, "Simulation based analysis of mobile sink speed in wireless sensor network," Proceeding of the World Congress on Engineering and Computer Science, Vol I, 2010.

[13] Ian D. Chakeres, Luke Klein-Verndt, "AODVjr, AODV simplified," Mobile Computing and Communication Review, pp.100-101. 2002.

[14] C. Gomez, P. Salvatella, O. Alonso, J. Paradells, "Adapting AODV for IEEE 802.15.4 mesh sensor networks: theoretical discussion and performance evaluation in a real environment," World of Wireless, Mobile and Multimedia Networks, pp. 170. 2006.

[15] Y. Xu, S.B. QIU, M. Hou, "Routing across bad node in the application of AODVjr local repair mechanism," Wireless Communications, Networking and Mobile Computing, 5th International Conference, pp. 1-4. 2009.
[16] F. Qiu, J. Wang, J. Leng, X. Xiao, "Design and implementation of a wireless personal area network based on AODVjr routing," Wireless, Mobile and Multimedia Networks, IET International Conference, pp. $1-4,2006$

[17] Shang Tao, Wu Wei, Liu Xu-Dong, Liu Jian-Wei, "AODVjr routing protocol with multiple feedback policy for ZigBee network," Consumer Electronics ISCE '09. pp. 483 - 487, 2009.

[18] C.Perkins, E. Belding-Royer, S. Das, "Ad hoc on-demand distance vector (AODV) routing," RFC 3561.

[19] T. Clausen, A. Colin de Verdiere, J. Yi, A. Niktash, Y. Igarashi, H. Satoh, U. Herberg, C. Lavenu, T. Lys, C.Perkins, J. Dean, "The lightweight on-demand ad hoc distance-vector routing protocol - next generation (LOADng), " https://tools.ietf.org/html/draft-clausen-llnloadng-06.

[20] T.Clausen, C.Dearlove, J.Dean, C.Adjih, "Generalized mobile ad hoc network (MANET) packet/message format," RFC 5444. 